\title{
Analytical solutions of the microscopic two-band theory for the temperature dependence of the upper critical fields of pure $\mathrm{MgB}_{2}$ compared with experimental data
}

\author{
M. Palistrant ${ }^{1}$, A. Surdu ${ }^{2}$, V. Ursu ${ }^{1}$, P. Petrenko ${ }^{1}$, and A. Sidorenko ${ }^{2}$ \\ ${ }^{1}$ Institute of Applied Physics ASM, Chisinau MD2028, Republic of Moldova \\ ${ }^{2}$ Institute of Electronic Engineering and Industrial Technologies ASM, Chisinau MD2028, Republic of Moldova \\ E-mail: andrey.su@yahoo.com
}

Received June 29, 2010, revised November 19, 2010

\begin{abstract}
Main theoretical results of the microscopic two-band theory for the temperature dependence of the upper critical fields $H_{c 2}(a b)$ and $H_{c 2}(c)$ in pure two-band systems like $\mathrm{MgB}_{2}$ are presented. The analytical solutions for the upper critical fields near the superconducting transition temperature and near the zero temperature were transformed to be directly compared with experimental data. The experimental $H_{c 2}(a b)$ and $H_{c 2}(c)$ temperature dependences of textured $\mathrm{MgB}_{2}$ films near the superconducting transition temperature were measured and compared with the respective theoretical formulas. The results of this theoretical approach were also compared with earlier published experimental data of other authors. The chosen method allows obtaining an accurate match between the theoretical expressions and experimental results.
\end{abstract}

PACS: 74.20.--Z Theories and models of superconducting state.

Keywords: superconductivity, microscopic two-band theory, analytical solutions, upper critical fields.

\section{Introduction}

The discovery of the high temperature superconductivity in the intermetallic compound $\mathrm{MgB}_{2}$ (with the critical temperature $T_{C} \sim 39 \mathrm{~K}$ ) led to an intensive use of a twoband model proposed by Moskalenko [1] and independently by Shul et al. [2].

This model assumes the existence of an overlap of various energy bands on the Fermi surface and, consequently, of the anisotropy of the electron energy spectrum, inherent in real superconducting systems. After publication of [1] and [2] a new line of research appeared, namely, the determination of the physical properties of two-band (or multiband) superconductors. Moldavian physicists headed by V. Moskalenko have brought a significant contribution to the development of this research direction. Many books and a lot of articles concerning this problem were published. Let us mention some publications related to the history of multiband superconductivity: books [3-6] reviews [7-9], and articles [10-12]. These works contain the names of scientists from various countries who have contributed to the development of the theory of two-band superconductivity. In particular, let us take note of reviews [13-15]. We can see from the above mentioned links that a lot of studies had been carried out long before the discov- ery of high temperature superconductivity and, moreover, before the discovery of superconductivity in $\mathrm{MgB}_{2}$. The two-band theory of superconductivity can explain many anomalies of the physical properties of real superconductors (see, for example, $[3,8,9]$ ) and it can be regarded as a classical theory. It is obvious that for any application it needs to be clarified, developed and generalized. It can be also applied for the case of $\mathrm{MgB}_{2}$, which can be considered as a two-band anisotropic superconductor, because the energy spectrum of the electrons in this compound is anisotropic, namely, there are two different energy bands on the Fermi surface: one band ( $\sigma$-band) is two-dimensional, while the other band ( $\pi$-band) is three-dimensional, which leads to the appearance of additional peculiarities in a number of physical characteristics of this compound (see [16-18]).

It is impossible to refer to all theoretical and experimental works that were carried out in order to determine the physical properties of $\mathrm{MgB}_{2}$. Let us note, in particular, that a two-band model with the variable density of charge carriers leads to a good agreement with experimental data for the thermodynamic and magnetic properties of $\mathrm{MgB}_{2}$ while replacing $\mathrm{Mg}$ and $\mathrm{B}$ with other elements of the periodic table $[19,20]$. 
For the development of the two-band theory of superconductivity (after publications $[1,2]$ ) it was also necessary to construct a microscopic theory for the upper critical field $\mathrm{Hc}_{2}$ for a pure two-band superconductor. Such a theory proposed for the first time in [21,22] appeared long before the discovery of superconductivity in $\mathrm{MgB}_{2}$. As a matter of fact, the anisotropy of the system required the construction of a new theory (in particular, for the magnetic properties of $\mathrm{MgB}_{2}$ ).

We are aware of several theoretical publications [23$26]$ in which the behavior of the upper critical field $H_{c 2}$ is described for a pure two-band superconductor with anisotropic properties similar to those of $\mathrm{MgB}_{2}$. In [23] and [24] an original theoretical approach was developed. For example, the value of $H_{c 2}$ in [23] is determined using a multiband formulation of the Elenberg semi-classical theory [27]. Owing to the strong anisotropy, the values of the upper critical fields, namely, the upper critical field in the $a b$-pane $H_{c 2}(a b)$ and the upper critical field in the $c$ axis direction $H_{c 2}(c)$, are considerably different which makes it possible to obtain results for pure $\mathrm{MgB}_{2}$ close to experimental data. It was shown in [24] that the ratio of $H_{c 2}^{(a b)} / H_{c 2}^{(c)}$ increases upon cooling.

In [25] and [26] the studies of the magnetic properties of the two-band superconductors were carried out using the phenomenological Ginzburg-Landau model applied to $\mathrm{MgB}_{2}$ and other compounds. A good agreement between theoretical and experimental data for the upper critical fields $H_{c 2}(a b)$ and $H_{c 2}(c)$ as functions of temperature was obtained. Note that we do not cite here papers regarding the $\mathrm{MgB}_{2}$ system with impurities. In our opinion, along with a phenomenological theory, it is also necessary to develop a microscopic theory for a two-band superconductor in a magnetic field. This theory is the next step leading to a deeper understanding of the basic properties of twoband superconductors and valid results.

The main purpose of this work is to construct a microscopic theory of the upper critical field $H_{C 2}$ in pure $\mathrm{MgB}_{2}$ based on the basic principles of the superconductor theory for a system in a magnetic field $[28,29]$ and to compare the obtained results with exact experimental data.

Below, we focus our attention mainly on the overlap of the energy bands on the Fermi surface (multiband case) and on the anisotropy due to different dimensions of the energy bands under study. This circumstance, in its turn, leads to different topologies of the Fermi surface cavities.

The studies below are based on a set of GinzburgLandau equations for the order parameters for a two-band system in a magnetic field [30,31]. The techniques of calculating of the $H_{c 2}$ values for anisotropic two-band systems are developed and generalized. The equations for $H_{c 2}$ and $T_{C}$ are derived, the asymptotic solutions for the $H_{c 2}$ value are found; the $H_{C 2}(T)$ curves are studied in the entire temperature range $0<T<T_{C}$ in anisotropic two-band systems.
Note that the regard for only one type of anisotropy (the overlap of the energy bands on the Fermi surface) [22] leads to an important, qualitatively new result in comparison with a single-band system; that is, in the $H_{c 2}$ dependence on $T$ there is a positive curvature that appears in the vicinity of the superconducting transition temperature. This fact results from the difference between the average velocities of the electrons on different cavities of the Fermi surface. Another anisotropy, caused by the inequivalence of the energy bands under study, leads to a significant difference between the $H_{c 2}(a b)$ value and the $H_{c 2}(c)$ value measured at the same temperature. In addition, the value of $H_{c 2}(a b)$ is several times greater than the $H_{c 2}(c)$ value. The two-dimensionality of the $\sigma$-band of $\mathrm{MgB}_{2}$ and small values of the average velocities of the electrons on the Fermi surface in the $c$-axis direction are the most important facts to be taken into account while solving this problem. In this work, we analyze the case of anisotropic systems with two different orientations of the applied magnetic field: $H \| a b$-plane and $H \| c$-axis.

In this work we present the analytical solutions of the equations for $H_{c 2}(a b)$ and $H_{c 2}(c)$ for the case of low temperatures $\left(T<<T_{C}\right)$ and near the superconducting transition temperature $\left(T_{C}-T<T_{C}\right)$. These solutions are transformed to a convenient form for comparison with experimental data, namely, we write them in the form of explicit functions of temperature and implicit functions of theory parameters. Such representation is useful while analyzing experimental data (the $H_{c 2}$ temperature dependence).

In this work, all theoretical results are presented briefly and concisely, they allow understanding the overall picture of occurring processes. For more details in addition to [8], see Ref. 22 , Refs. 32-35, and review article [36]. It follows from these works that the temperature dependences of $H_{c 2}(a b)$ and $H_{c 2}(c)$ are consistent with theoretical results [23-26] and available experimental data.

In the experimental part of this work, we compare the theory presented in this paper with our experimental results and with results of other authors on the $H_{c 2}(a b)$ and $H_{c 2}(c)$ temperature dependences.

\section{Theory}

The current study is based on a set of the GinzburgLandau equations for the order parameters $\Delta_{m}(\mathbf{x})$ in a magnetic field [30]; we consider the case of high values of a magnetic field $H$ (close to the value of the upper critical field $H_{C 2}$ ), i.e., the subcritical region in the vicinity of the unstable normal state. In this case it is possible to keep the linear terms of the order parameters $\Delta_{m}^{*}(m=1 ; 2)$ in the set of the Ginzburg-Landau equations.

On this account, we obtain:

$$
\Delta_{m}^{*}(\mathbf{r})=\frac{1}{\beta} \sum_{n} V_{n m} \sum_{\omega} \int d \mathbf{r}^{\prime} g_{n}\left(\mathbf{r}, \mathbf{r}^{\prime}, \omega\right) \Delta_{n}^{*}(\mathbf{r}) g_{n}\left(\mathbf{r}, \mathbf{r}^{\prime},-\omega\right)
$$


In the presence of a magnetic field, the electronic Green function $g_{n}$ is defined by the following expression [28]:

$$
g_{n}\left(\mathbf{r}, \mathbf{r}^{\prime}, \omega\right)=\mathrm{e}^{i \varphi\left(\mathbf{r}, \mathbf{r}^{\prime}\right)} g_{n}^{0}\left(\mathbf{r}, \mathbf{r}^{\prime}, \omega\right) .
$$

Here $g_{n}^{0}$ is the Green function in the normal state without any magnetic field. The presence of a magnetic field is taken into account by the phase multiplier:

$$
\varphi\left(\mathbf{r}^{\prime}, \mathbf{r}\right)=\int_{\mathbf{r}}^{\mathbf{r}^{\prime}} A(\mathbf{l}) d \mathbf{l} .
$$

We shall substitute definition (2) in (1), performing expansion of the function $g_{n}^{0}$ in terms of the Bloch functions; after that, we shall average this equation over the amplitudes of the Bloch functions. In this way, we obtain:

$$
\begin{gathered}
\Delta_{m}^{*}(\mathbf{r})=\sum_{n} V_{n m} \frac{1}{\beta} \sum_{\omega} \int d \mathbf{r} \sum_{\mathbf{k}} \sum_{\mathbf{q}} g_{n}^{0}(\mathbf{k}, \omega) \times \\
g_{n}^{0}(\mathbf{q}-\mathbf{k},-\omega) \Delta_{n}^{*}\left(\mathbf{r}^{\prime}\right) \mathrm{e}^{2 i \varphi\left(\mathbf{r}^{\prime}, \mathbf{r}\right)} \mathrm{e}^{i \mathbf{q}\left(\mathbf{r}^{\prime}-\mathbf{r}\right)} \\
g_{n}^{0}(\mathbf{k}, \omega)=\left[i \omega-\xi_{n}(\mathbf{k})\right]^{-1}
\end{gathered}
$$

where $\omega$ is the Matsubar frequency, $\xi_{n}$ is the electron energy in the $n$th band.

\subsection{Calculation of the temperature dependence of the upper critical field parallel to the ab-plane}

Herein, it is possible to choose:

$A_{z}=-\left(H_{0} / 2\right)\left(x+x^{\prime}\right), A_{y}=A_{x}=0$ in the symmetric view and based on (3) we obtain:

$$
2 \varphi\left(\mathbf{r}^{\prime}, \mathbf{r}\right)=e H_{0}\left(x+x^{\prime}\right)\left(z-z^{\prime}\right) .
$$

We shall represent the dispersion law for the $\sigma$ - and $\pi$ bands (it is singed by index 1 and 2 , respectively) in the following form:

$$
\begin{aligned}
& \xi_{1}(\mathbf{k})=\zeta_{1}+\frac{k_{x}^{2}+k_{y}^{2}}{2 m_{1}}+\frac{k_{z}^{2}}{2 M}-\mu, \\
& \xi_{2}(\mathbf{k})=\zeta_{2}+\frac{k_{x}^{2}+k_{y}^{2}+k_{z}^{2}}{2 m_{2}}-\mu,
\end{aligned}
$$

where $M \gg>m_{1}$. The satisfaction of this inequality leads to a weak deviation of the dispersion law of the first band from two-dimensionality. The Fermi surface of the other band is assumed to be spherical for simplicity.

Subsequently, Eq. (4) is transformed using generalized techniques of Maki and Tsuzuki [29] for the case of a twoband anisotropic superconductor with electron energy dispersion law (7). Note that technique [29] contains two main parts: the execution, if possible, of all operations on the right-hand side of Eq. (4) (for details see [36], Appen$\operatorname{dix} \mathrm{A})$ and the execution of a number of mathematical transformations in terms of the microscopic theory of superconductivity [34-36]. In this way, set of Eq. (1) can be reduced to:

$$
\begin{aligned}
& \Delta_{1}^{*}=\lambda_{11} \Delta_{1}^{*}\left[\xi^{(1)}(T)-f_{11}\left(\rho_{1} \tilde{\varepsilon}\right)\right]+\tilde{\lambda}_{12} \Delta_{2}^{*}\left[\xi^{(2)}(T)-f_{12}\left(\rho_{2} \tilde{\varepsilon}\right)\right] \\
& \Delta_{2}^{*}=\lambda_{21} \Delta_{1}^{*}\left[\xi^{(1)}(T)-f_{21}\left(\rho_{1} \tilde{\varepsilon}\right)\right]+\tilde{\lambda}_{22} \Delta_{2}^{*}\left[\xi^{(2)}(T)-f_{21}\left(\rho_{2} \tilde{\varepsilon}\right)\right]
\end{aligned}
$$

where

$$
\begin{gathered}
\xi^{(n)}(T)=\int_{-d_{n}}^{d_{c n}} d \varepsilon \frac{\operatorname{th}(\beta \varepsilon / 2)}{2 \varepsilon}, \quad \tilde{\lambda}_{12}=\tilde{\varepsilon}^{1 / 2} \sqrt{\frac{2}{\tilde{\varepsilon}+1}} \lambda_{12} \\
\tilde{\lambda}_{21}=\sqrt{\frac{2}{\tilde{\varepsilon}+1}} \lambda_{21},
\end{gathered}
$$

where $\beta=1 / T$, and the values $d_{n}=\mu-\zeta_{n}, d_{c n}=\zeta_{c n}-\mu$ are the parameters of the integrals taken over the energy at the variable density of charge carriers, $\tilde{\varepsilon}$ is the small parameter, which determines a deviation of the $\sigma$-band from the two-dimensionality. Under the phonon mechanism of superconductivity (the case of $\mathrm{MgB}_{2}$ ) these parameters have the following form:

$$
\begin{gathered}
d_{n}=\left\{\begin{array}{lll}
\mu-\zeta_{n} & \text { at } & \mu-\zeta_{n} \leq \omega_{D}^{(n)}, \\
\omega_{D}^{(n)} & \text { at } & \mu-\zeta_{n}>\omega_{D}^{(n)},
\end{array}\right. \\
d_{c n}=\left\{\begin{array}{ccc}
\omega_{D}^{(n)} & \text { at } & \zeta_{c n}-\mu>\omega_{D}^{(n)}, \\
\zeta_{c n}-\mu & \text { at } & \zeta_{c n}-\mu<\omega_{D}^{(n)} .
\end{array}\right.
\end{gathered}
$$

Here $\omega_{D}^{(n)}$ is the characteristic phonon frequency corresponding to the $n$th energy band. The functions fnm containing the dependence on a magnetic field can be written as follows:

$$
\begin{gathered}
f_{11}=\frac{\left(\tilde{\varepsilon} \rho_{1}\right)^{-1 / 2}}{\pi} \int_{-1}^{1} d y \int_{1}^{\infty} \frac{d u}{\sqrt{u^{2}-1}} \int_{0}^{\infty} \frac{d \zeta}{\operatorname{sh}\left(\zeta u /\left(\tilde{\varepsilon} \rho_{1}\right)^{1 / 2}\right)}\left\{1-\exp \left[\left(-\zeta^{2} / 2\right)\left(1-u^{2} y^{2}\right)\right]\right\}, \\
f_{12}=\rho_{2}-1 / 2 \int_{0}^{\pi} d \varphi \int_{1}^{\infty} \frac{d u}{u} \int_{0}^{\infty} \frac{d \zeta}{\operatorname{sh}\left(\zeta \mathrm{u} / \rho_{2}^{1 / 2}\right)}\left\{1-\exp \frac{-\zeta^{2}(1+\tilde{\varepsilon})}{4}\left[1-\left(\frac{\tilde{\varepsilon}-1+2 i \sqrt{u^{2}-1} \cos \varphi}{1+\tilde{\varepsilon}}\right)^{2}\right]\right\},
\end{gathered}
$$




$$
\begin{gathered}
f_{21}=\frac{\rho_{1}^{-1 / 2}}{\pi} \int_{-1}^{1} d y \int_{1}^{\infty} \frac{d u}{\sqrt{u^{2}-1}} \int_{0}^{\infty} \frac{d \zeta}{\operatorname{sh}\left(\zeta u / \rho_{1}^{1 / 2}\right)}\left\{1-\exp \frac{-\zeta^{2}(1+\tilde{\varepsilon})}{4}\left[1-\left(\frac{1-\tilde{\varepsilon}-2 i \tilde{\varepsilon} u y}{1+\tilde{\varepsilon}}\right)^{2}\right]\right\}, \\
f_{22}=\rho_{2}{ }^{-1 / 2} \int_{1}^{\infty} \frac{d u}{u} \int_{0}^{\infty} \frac{d \zeta}{\operatorname{sh}\left(\zeta u / \rho_{2}^{1 / 2}\right)}\left[1-\exp \frac{-\zeta^{2}\left(1+u^{2}\right)}{4} I_{0}\left(\frac{\zeta^{2}\left(u^{2}+1\right)}{4}\right)\right] .
\end{gathered}
$$

The dimensionless parameter $\rho_{2}^{-1 / 2}=\left[v_{n}\left(e H_{0}\right)^{1 / 2}\right] / 2 \pi T$ contains the value of $H_{0}=H_{c 2}^{(a b)}$, it was introduced in the definition of the functions $f_{n m} ; v_{n}$ is the velocity of the electrons on the $n$th cavity of the Fermi surface, $I_{0}$ is the Bessel function of the imaginary argument.

From a condition of solvability of set (8) at $H \rightarrow \infty$, we obtain an equation for the superconducting transition temperature $T_{C}$ :

$$
a \xi^{(1)}\left(T_{C}\right) \xi^{(2)}\left(T_{C}\right)-\lambda_{11} \xi^{(1)}\left(T_{C}\right)-\lambda_{22} \xi^{(2)}\left(T_{C}\right)+1=0,
$$

where $a=\lambda_{11} \lambda_{22}-\lambda_{12} \lambda_{21}$, and $\xi^{(n)}(T)$ is given by formula (9). Equating the determinant of set (8) to zero and using Eq. (15), we obtain the equation for the determination of the upper critical field $H_{0}=H_{c 2}^{(a b)}$ when the superconducting pairs appear. This equation has the form:

$$
\begin{gathered}
\lambda_{11} \lambda_{22} \tilde{F}_{11} \tilde{F}_{22}-\lambda_{12} \lambda_{21} \tilde{F}_{12} \tilde{F}_{21}+\lambda_{11}\left[1-\lambda_{22} \xi^{(2)}\left(T_{C}\right)\right] \tilde{F}_{11}+ \\
\left.+\lambda_{22}\left[1-\lambda_{11} \xi^{(1)}\left(T_{C}\right)\right] \tilde{F}_{22}+\lambda_{12} \lambda_{21} \xi^{(2)}\left(T_{c}\right)\right] \tilde{F}_{21}+ \\
\left.+\lambda_{12} \lambda_{21} \xi^{(1)}\left(T_{C}\right)\right] \tilde{F}_{12}=0
\end{gathered}
$$

where

$$
\tilde{F}_{m n}=f_{m n}+\ln T / T_{c}, \quad f_{m n}=f_{m n}\left(\rho_{n}, \tilde{\varepsilon}\right) .
$$

Equation (16) contains complex integral dependences $f_{m n}(11)-(14)$; it is possible to solve this equation in the entire temperature interval $0<T<T_{C}$ only by numerical methods. However, it is possible to find its analytical solutions for two limit cases: a) in the vicinity of the critical temperature $\rho_{n}<<1$, when $T_{C}-T<<T_{C}$ and $b$ ) in the range of low temperatures $\rho_{n}>>1$ at $T<<T_{c}$. For each of these cases we obtain asymptotic expressions for functions fnm. We shall substitute the values of these functions into (16) with some preliminary simplifications (for details see [34] and Appendix B in [36]). Thereupon, we obtain:

$$
\begin{gathered}
\text { a) } T_{C}-T<<T_{C}, \quad \rho_{n}<<1: \\
\rho_{C}^{0}=\frac{v_{1} v_{2} e H_{0}(T)}{\left(2 \pi T_{C}\right)^{2}}=\left[\frac{v \theta}{\tilde{\beta}}+\frac{v\left(\tilde{\beta}^{2}-2 \tilde{\alpha} v\right) \theta^{2}}{2 \tilde{\beta}^{2}}\right] \lambda^{-1}\left(\frac{T}{T_{C}}\right)^{2}
\end{gathered}
$$

where

$$
\theta=1-\frac{T}{T_{C}}, \lambda=\frac{v_{1}}{v_{2}}, \text { and } v=\sqrt{\left(\lambda_{11}-\lambda_{22}\right)^{2}+4 \tilde{\lambda}_{12} \tilde{\lambda}_{21}}
$$

$$
\begin{gathered}
\tilde{a}=-\frac{31}{10} \zeta(5)\left[\frac{\lambda_{22}-a \xi_{c}}{\lambda^{4}}-\frac{\tilde{\varepsilon}^{2}}{16} \xi_{c}\left(\frac{109}{4} \lambda_{11} \lambda_{22}-\right.\right. \\
\left.\left.-\frac{25+80 \tilde{\varepsilon}+4 \tilde{\varepsilon}^{2}}{(1+\tilde{\varepsilon})^{2}} \tilde{\lambda}_{12} \tilde{\lambda}_{21}\right)+\frac{109}{64} \tilde{\varepsilon}^{2} \lambda_{11}\right], \\
\tilde{\beta}=\frac{7}{6} \zeta(3)\left[\frac{\lambda_{22}-a \xi_{c}}{\lambda^{2}}-\frac{\tilde{\varepsilon}}{2} \xi_{c}\left(\frac{5}{2} \lambda_{11} \lambda_{22}-\right.\right. \\
\left.\left.-\frac{(3+2 \tilde{\varepsilon})}{2(1+\tilde{\varepsilon})} \tilde{\lambda}_{12} \tilde{\lambda}_{21}\right)+\frac{5}{4} \tilde{\varepsilon} \lambda_{11}\right] .
\end{gathered}
$$

Expression (18) can be written as:

$$
H_{0}(T)=H_{c 2}(T)=æ_{1}\left(\theta+æ_{2} \theta^{2}\right) T^{2},
$$

where

$$
æ_{1}=\frac{4 \pi^{2} v}{e v_{1}^{2} \widetilde{\beta}}, \quad æ_{2}=\left(\tilde{\beta}^{2}-2 \tilde{\alpha} v\right) / 2 \tilde{\beta}^{3}
$$

It follows from (20) and (22) that the temperature independent coefficients $æ_{1}$ and $æ_{2}$ are the composite functions of the theory parameters $\left(\lambda_{n m}, \tilde{\varepsilon}\right)$.

b) At low temperatures $T<<T_{C}$ and $\tilde{\varepsilon} \rho_{n} \gg 1$ the solution to Eq. (16) determining the value of $H_{C 2}(T)$ has the form:

$$
H_{c 2}(T)=H_{c 2}(0)\left[1-\frac{2 F(T)}{\sqrt{\bar{B}^{2}-4 a \bar{C}}}\right]
$$

where

$$
H_{c 2}(0)=\frac{\left(2 \pi T_{c}\right)^{2}}{v_{1} v_{2} e}(\gamma \tilde{\varepsilon})^{-1} \exp \left[\frac{-\bar{B} \pm \sqrt{\bar{B}^{2}-4 a \bar{C}}}{a}\right],
$$

$$
\begin{aligned}
& a=\lambda_{11} \lambda_{22}-\tilde{\lambda}_{12} \tilde{\lambda}_{21}, \quad \bar{B}=0,038 a+v-\Lambda \\
& \bar{C}=\Lambda \xi_{c}-a \ln \lambda+\ln [\sqrt{\lambda]}(0.04-a \ln [\sqrt{\tilde{\varepsilon}}]+ \\
& \left.+\lambda_{11}-\lambda_{22}+\Lambda\right)+0.11 \lambda_{11}-(0.4-a \ln [\sqrt{\tilde{\varepsilon}}])\left(\Lambda+a \xi_{c}-\lambda_{22}\right) \\
& \Lambda=\ln [c(\tilde{\varepsilon})] \tilde{\lambda}_{12} \tilde{\lambda}_{21}-0.11 \lambda_{11} \lambda_{22} \\
& \ln [c(\tilde{\varepsilon})]=0.235+\frac{1}{2} \ln \frac{1+\sqrt{1+\tilde{\varepsilon}}}{1+\tilde{\varepsilon}}+\frac{1}{\tilde{\varepsilon}} \sqrt{1+\frac{1}{\tilde{\varepsilon}^{2}}}
\end{aligned}
$$


At the same time $F(T)$ is defined by the following expression:

$$
F(T)=\chi_{1} t^{2}+\chi_{2} t^{2} \ln t,
$$

where

$$
t=T / T_{c}
$$

$$
\begin{aligned}
& \chi_{1}=A_{11} a_{11}-A_{22} a_{22}+A_{12} a_{12}-A_{21} a_{21}, \\
& \chi_{2}=A_{11} b_{11}-A_{22} b_{22}+A_{12} b_{12}-A_{21} b_{21} \text {, } \\
& A_{11}=\left[\tilde{\lambda}_{12} \tilde{\lambda}_{21} \xi_{c}-\ln (x c(\tilde{\varepsilon}) \sqrt{\lambda})\right] \frac{\lambda}{\pi^{2} \rho_{c 0}^{0}}, \\
& A_{22}=\left[\ln \left(\frac{2 \sqrt{2} x}{e_{0} \sqrt{\lambda \tilde{\varepsilon}}}\right)-\xi_{c}\right] \frac{1+\tilde{\varepsilon}}{2 \pi^{2} \rho_{c 0}^{0} \lambda \tilde{\varepsilon}^{3 / 2}}, \\
& A_{12}=\lambda_{22}\left(\xi_{c}-\lambda_{11} \ln (x c(\tilde{\varepsilon}) \sqrt{\lambda})-1\right) \frac{\lambda}{\pi^{2} \rho_{c 0}^{0}}, \\
& A_{21}=\left\{\lambda_{11} \lambda_{22}\left[\ln \left(\frac{2 \sqrt{2} x}{e_{0} \sqrt{\lambda \tilde{\varepsilon}}}\right)+\xi_{c}-\lambda_{11}\right]\right\} \frac{1}{\pi^{2} \rho_{c 0}^{0} \lambda \tilde{\varepsilon}}, \\
& a_{11}=\varsigma^{\prime}(2), \quad a_{22}=\varsigma^{\prime}(2)+\frac{\varsigma(2) \tilde{\varepsilon}}{4}, \\
& a_{12}=-\varsigma^{\prime}(2), \quad a_{21}=-\varsigma^{\prime}(2), \\
& b_{11}=-\frac{\varsigma(2)}{2} \ln \frac{\rho_{c 0}^{0} \gamma \pi^{2}(1+\tilde{\varepsilon})}{4 \lambda}, \\
& b_{22}=-\frac{\varsigma(2)}{2} \ln \frac{\tilde{\varepsilon}^{2} \rho_{c 0}^{0} \gamma \pi^{2} \lambda}{1+\tilde{\varepsilon}}, \\
& b_{12}=\frac{\varsigma(2)}{2} \ln \frac{\rho_{c 0}^{0} \gamma \pi^{2}}{2 \lambda}, b_{21}=\frac{\varsigma(2)}{2} \ln \frac{\rho_{c 0}^{0} \gamma \pi^{2} \tilde{\varepsilon} \lambda}{2 e_{0}^{2}} \text {, } \\
& x=\sqrt{\tilde{\varepsilon} \gamma \rho_{c 0}^{0}}, \quad \rho_{c 0}^{0}=\frac{v_{1} v_{2} e H_{0}(0)}{\left(2 \pi T_{c}\right)^{2}} .
\end{aligned}
$$

Substituting expression (25) in (23) we obtain the following formula for the temperature dependence of the upper critical field:

$$
H_{c 2}(T)=H_{c 2}(0)\left[1-d_{1} t^{2}-d_{2} t^{2} \ln t\right] .
$$

Here

$$
d_{i}=2 \chi_{i} / \sqrt{\bar{B}^{2}-4 a \bar{C}}, \quad i=1,2 .
$$

As it follows from definitions (26)-(30), the coefficients $d_{1}$ and $d^{2}$ are the composite functions of theory parameters.

\subsection{Calculation of the temperature dependence of the upper critical field parallel to the c-axis}

Above we have developed the theory of the upper critical field $H_{c 2}(a b)$ for a two-band anisotropic system. In $\mathrm{MgB}_{2}$ this is the highest possible value of the upper critical field. It is of interest to adduce the results of $H_{c 2}(c)$ calculation which corresponds to the smallest value of the upper critical field in $\mathrm{MgB}_{2}$ for the case of a magnetic field parallel to the $c$-axis direction. The both results allow obtaining the temperature dependence of the anisotropy coefficient $\gamma_{H}=H_{c 2}^{(a b)} / H_{c 2}^{(c)}$ for the upper critical fields.

Let us consider $\mathbf{H} \| \mathbf{c}$. In this case, it is possible to choose $A_{x}=A_{z}=0, A_{y}=H_{0}\left(x+x^{\prime}\right) / 2$, and we obtain for the phase multiplier the following expression:

$$
2 \varphi\left(\mathbf{r}^{\prime}, \mathbf{r}\right)=e H_{0}\left(x+x^{\prime}\right)\left(y-y^{\prime}\right) .
$$

For the case of $\mathbf{H} \| \mathbf{c}$, the average velocity of the electrons in the $a b$-plane plays an important role for either energy band, while the value of the electron velocity in the $z$-direction is negligible. Therefore, it is unnecessary to introduce a parameter controlling the deviation of the $\sigma$ band from the 2D behavior making the problem less anisotropic (for details see [36]).

Let us consider some results. The critical magnetic field $H_{C 2}(c)$ is defined by the following equation:

$$
\begin{gathered}
a \tilde{f}_{1}\left(\rho_{1}\right) \tilde{f}_{2}\left(\rho_{2}\right)+\left[\lambda_{11}-a \xi_{c}\right] \tilde{f}_{1}\left(\rho_{1}\right)+ \\
+\left[\lambda_{22}-a \xi_{c}\right] \tilde{f}_{2}\left(\rho_{2}\right)=0,
\end{gathered}
$$

where

$$
\begin{gathered}
\tilde{f}_{1}\left(\rho_{1}\right)=f_{1}\left(\rho_{1}\right)-\ln \left(\frac{T_{c}}{T}\right)^{1 / 2}, \\
\tilde{f}_{2}\left(\rho_{2}\right)=f_{2}\left(\rho_{2}\right)-\ln \frac{T_{C}}{T} . \\
f_{1}\left(\rho_{1}\right)=\frac{\rho_{1}^{-1 / 2}}{\pi} \int_{1}^{\infty} \frac{d u}{\sqrt{u^{2}-1}} \times \\
\times \int_{0}^{\infty} \frac{d \zeta}{\operatorname{sh}\left(\zeta u / \rho_{1}^{1 / 2}\right)}\left(1-\exp \left(-\zeta^{2} u^{2} / 2\right)\right) .
\end{gathered}
$$

The function $f_{2}\left(\rho_{2}\right)$ corresponds to the expression for $f_{22}(14)$.

Here, as we have done in Sec. 2.2, we can the analytical solutions of Eq. (33) by substituting in (34) the asymptotic values of the functions $\tilde{f}_{n}$ in the following range of values (a) $T_{C}-T<<T_{C}, \rho_{n}<<1$ and (b) $T<<T_{C}, \rho_{n}>1$ and keeping the terms with the core contribution, see $[33,36]$. These solutions can be represented as follows:

a) At temperatures near the critical $\left(T_{C}-T<<T_{C}\right)$ we obtain the following formula for the temperature dependence of the upper critical field: 


$$
H_{c 2}(T)=A_{1} \theta+A_{2} \theta^{2}
$$

where

$$
\begin{gathered}
A_{1}=\frac{\left(2 \pi T_{c}\right)^{2}}{v_{1}^{2} e} \alpha_{1}, \quad A_{2}=\left(2 \pi T_{c}\right)^{2} \frac{\alpha_{2}}{v_{1}^{2}} e, \\
\alpha_{1}=\frac{\eta_{1}+2 \eta_{2}}{7 \zeta(3)\left[\frac{\eta_{1}}{4}+\frac{\eta_{2}}{3} \frac{1}{\lambda^{2}}\right]}, \\
\alpha_{2}=\alpha_{1}\left\{\frac{\left[\frac{31 \cdot 3}{16} \eta_{1}+\frac{31}{5} \frac{1}{\lambda^{2}} \eta_{2}\right] \varsigma(5) \alpha_{1}}{\left[\frac{1}{4} \eta_{1}+\frac{1}{3} \frac{1}{\lambda^{2}} \eta_{2}\right] 7 \varsigma(3)}+1\right\} . \\
\theta=1-T / T_{c}, \quad \eta_{1,2}=\frac{1}{2}[1 \pm \eta], \\
\eta=\left[\left(\lambda_{11}-\lambda_{22}\right)^{2}+4 \lambda_{11} \lambda_{22}\right]^{-1 / 2}\left[\lambda_{11}-\lambda_{22}\right] .
\end{gathered}
$$

b) at low temperatures $\left(T<<T_{c}\right)$ :

$$
H_{c 2}(T)=H_{c 2}(0)\left[1-\frac{F_{\mathrm{c}}(T)}{\Omega(\lambda)}\right],
$$

where

$$
\begin{gathered}
H_{c 2}(0)=\frac{\left(2 \pi T_{c}\right)^{2}}{v_{1} v_{2} e} \frac{e_{0}}{4 \gamma} \exp \left(\frac{\eta_{-}}{2}-\frac{3 v(1)}{2}+\Omega(\lambda)\right), \\
\Omega(\lambda)=\left\{\left[-\ln \left(\frac{e_{0} \lambda}{2}\right)+\frac{3}{2} \eta_{-}-\frac{1}{2} v(1)\right]^{2}+\frac{8 \lambda_{12} \lambda_{21}}{a^{2}}\right\}^{1 / 2},(40) \\
F_{c}(T)=\left(\frac{T}{T_{c}}\right)^{2}\left\{\frac{3}{2 \pi^{2}} \varsigma(2) \frac{1}{\rho_{1 c}^{0}}\left[v(1) \eta_{1}+\tilde{f}_{2}\left(\rho_{1 c}^{0}\right)\right]-\right. \\
\left.-\frac{1}{\pi^{2} \rho_{2 c}^{0}}\left[\varsigma^{\prime}(2)+\varsigma(2) \ln \left(\frac{2 T_{c}}{\pi^{2} \gamma \rho_{2 c}^{0} T}\right)\right]\left[v(1) \eta_{2}+\tilde{f}_{1}\left(\rho_{1 c}^{0}\right)\right]\right\},
\end{gathered}
$$

where $e_{0}$ is the base of the natural logarithm, $e$ is the electron charge.

Expression (38) can be written as follows:

$$
H_{c 2}(T)=H_{c 2}(0)\left[1-c_{1} t^{2}-c_{2} t^{2} \ln t\right] .
$$

It is easy to determine the coefficients $c_{1}$ and $c_{2}$ using Eqs. (39)-(41).

It follows from the above written analytical formulas for the upper critical fields at low temperatures and near $T_{C}$ that we have a significant dependence of the upper critical fields $H_{c 2}(a b)$ and $H_{c 2}(c)$ on the anisotropy of the electron energy spectrum and on other theory parameters. If we use the values of $\lambda_{n m}, v_{1}, v_{2}$ and other theory parameters from [19] and [20] in order to compare this theoretical approach with experiments on $\mathrm{MgB}_{2}$, we will obtain a qualitative agreement between them [32-36]; this can be explained, in particular, by inexact determination of the theory parameters (the spread of these values in the literature is quite substantial). In this work we transform the expressions for $H_{c 2}(a b)$ and $H_{c 2}(c)$ in the studied temperature range introducing coefficients at all temperature dependent terms (see, e.g., (21)). These coefficients are composite functions of the theory parameters. Experimental temperature dependence of the upper critical fields allows us to calculate the exact values of these coefficients. Therefore, if we know the exact values of the real theory parameters, we can obtain (see below) the full agreement of the microscopic twoband theory with experimental data for the temperature dependence of the upper critical fields in an anisotropic two-band superconductor.

\section{Experimental}

In order to compare the analytical expressions of the above presented theory with experiment, $\mathrm{MgB}_{2}$ films with a thickness of about $600 \mathrm{~nm}$ were prepared on the $c$-plane sapphire substrates using a "two-step" synthesis technology similar to the method described in detail in [37]. Aluminum wires with a diameter of $20 \mu \mathrm{m}$ were attached to the samples by ultrasonic bonding for four-probe resistance measurements using a DC technique. The total electric current through samples was $100-150 \mu \mathrm{A}$. The contact resistance was about $0.3 \mathrm{Ohm}$. The effect of the measuring current was negligible. The resistance measurements were performed at various magnetic fields oriented parallel (in Fig. 1) and perpendicular (in Fig. 2) to the sample surface using a 'Sumitomo F-50' closed-cycle ${ }^{4} \mathrm{He}$ refrigerator with an $8 \mathrm{~T}$ superconducting solenoid. All measurements

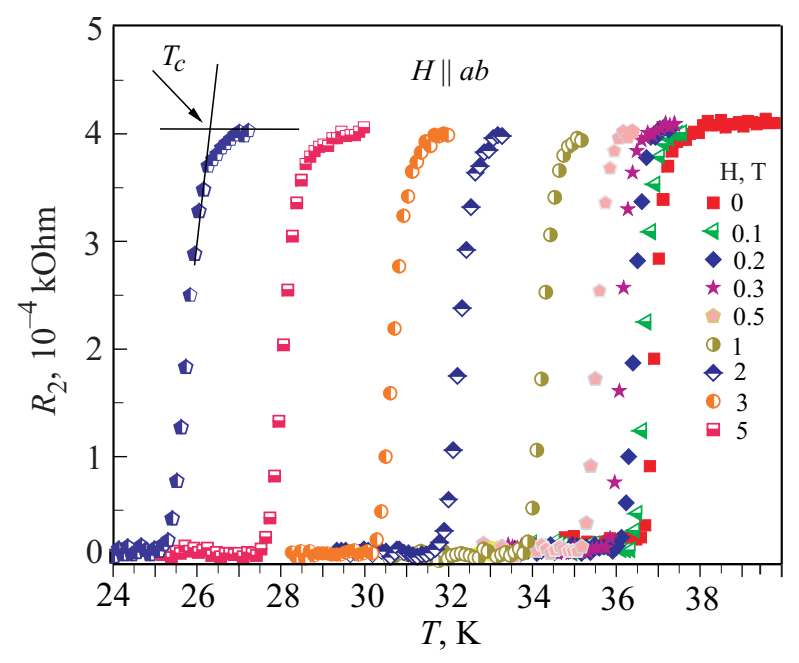

Fig. 1. Resistive transitions at various magnetic fields parallel to the surface of the $c$-axis oriented $\mathrm{MgB}_{2}$ film grown on the $c$-plane sapphire. 


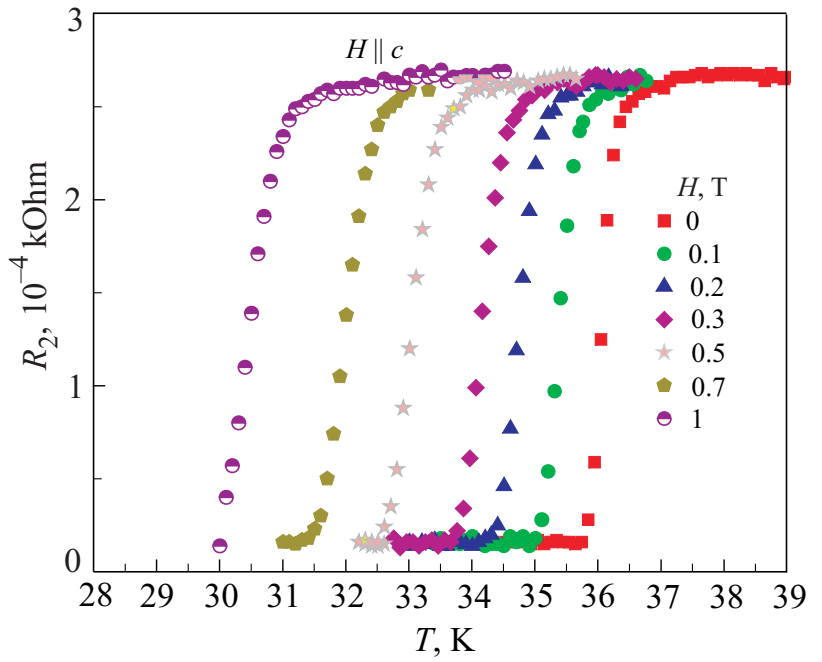

Fig. 2. Resistive transitions at various magnetic fields perpendicular to the surface of the $c$-axis oriented $\mathrm{MgB}_{2}$ film grown on the $c$-plane sapphire.

were carried out after 'zero field cooling' of the sample providing the absence of a remanent magnetization. The critical temperatures $T_{C}\left(H_{C 2}\right)$ were determined from the onset-points of the $R(T)_{H}=$ const curves. Using the obtained number of $T_{C}\left(H_{C 2}\right)$ points, we have plotted the $H_{C 2}(T)$ curve.

\section{Results and discussion}

The deposited phase composition was determined by the $\mathrm{x}$-ray diffraction (XRD) analysis using a DRON-UM diffractometer $\left(\theta-2 \theta\right.$ spectra, Fe $K_{\alpha}$ radiation). The XRD pattern consists (Fig. 3) of strong reflections (00l) from the single-crystal substrate $\alpha-\mathrm{Al}_{2} \mathrm{O}_{3}$ (c-plane sapphire) $[38,39]$ and the pronounced reflections of the $\mathrm{MgB}_{2}$ film.

The $\mathrm{x}$-ray peak intensity distribution differs from that of conventional $\mathrm{MgB}_{2}[38,39]$. This fact can be explained by the existence of a preferential orientation of crystallites along (001), i.e,. by the formation of a texture in the $\mathrm{MgB}_{2}$

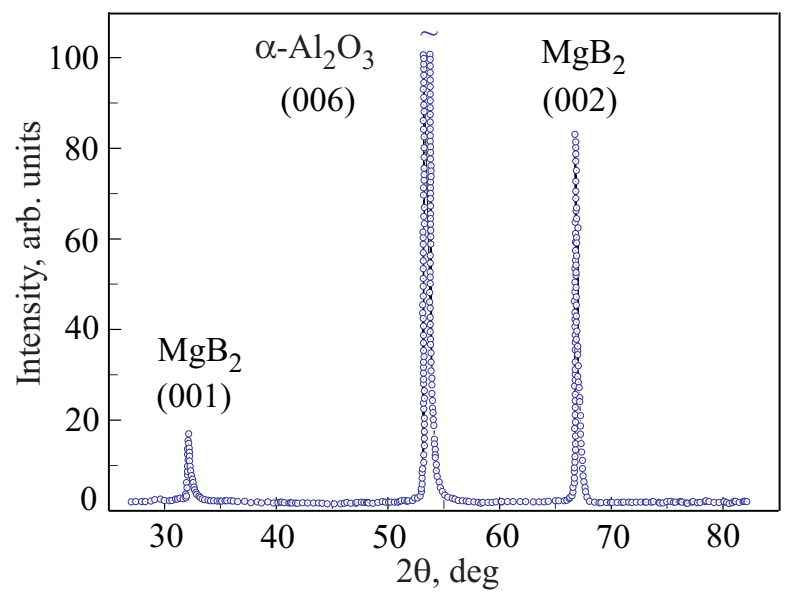

Fig. 3. X-ray diffraction pattern of a $\mathrm{MgB}_{2}$ film grown on the $c$ plane sapphire substrate. film. This texture $\tau(001)$ is parallel to the film plane. The coherent scattering region (the size of crystallites) calculated on the basis of the halfwidth of the $\mathrm{MgB}_{2}(001)$ and (002) diffraction peaks is $\sim 35-45 \mathrm{~nm}$. The $c$-axis parameter was found to be $0.3517(5) \mathrm{nm}$ for $\mathrm{MgB}_{2}$. According to the ASTM standard [38,39] $c=0.3522(2) \mathrm{nm}$.

Resistive transitions at various magnetic fields oriented parallel and perpendicular to the surface of this film are shown in Fig. 1 and Fig. 2, respectively. The critical temperature of the sample $T_{C}$ is $36.5 \mathrm{~K}$, the width of the transition is $0.3 \mathrm{~K}$ (at zero field). The RRR value is 1.75 . (RRR is the residual resistance ratio: $\left.R(300 \mathrm{~K}) / R\left(T_{C}\right)\right)$.

As we can see from Figs. 1, 2, and 3, this sample is of a high quality since its XRD reflections are very narrow and its resistive transitions are very sharp even in high fields. This fact demonstrates that the broadening of the superconducting transition of $\mathrm{MgB}_{2}$ in high fields, which was observed in resistivity measurements [40,41], can be caused not only by the inhomogeneity of samples but also by their polycrystalline structure. As a matter of fact, all grains that are tilted from a preferential orientation of the sample turn to the normal state before the others, which are oriented preferentially, because of the strong anisotropy of $\mathrm{MgB}_{2}$ in high fields, and suppress superconductivity in their vicinity due to the proximity effect, which creates a natural non-uniformity of the whole sample caused by anisotropy.

In order to compare Eq. (21) with the respective experimental temperature dependence of the upper critical field, let us calculate the coefficients $æ_{1}$ and $æ_{2}$ using only two experimental points from this experiment:

$$
\begin{gathered}
T_{1}=35.15 \mathrm{~K}, \quad \mu_{0} H_{0}\left(T_{1}\right)=0.5 \mathrm{~T} \\
\text { and } T_{2}=32.08 \mathrm{~K}, \mu_{0} H_{0}\left(T_{2}\right)=2 \mathrm{~T},
\end{gathered}
$$

where $\mu_{0}$ is the magnetic constant (vacuum permeability). By substituting these values into Eq. (21), we obtain a set of two equations:

$$
\begin{aligned}
& \mu_{0} H_{0}\left(T_{1}\right)=\mu_{0} æ_{1}\left(\theta_{1}+æ_{2} \theta_{1}^{2}\right) T_{1}^{2}, \\
& \mu_{0} H_{0}\left(T_{2}\right)=\mu_{0} æ_{1}\left(\theta_{2}+æ_{2} \theta_{2}^{2}\right) T_{2}^{2} .
\end{aligned}
$$

By solving this set of equations, we obtain:

$$
\mu_{0} æ_{1}=0.0085 \text { and } \mathfrak{x}_{2}=7.36
$$

Performing the same calculations for Eq. (37) we can obtain the coefficients:

$$
\mu_{0} A_{1}=5.3 \text { and } \mu_{0} A_{2}=9.16 \text {. }
$$

The temperature dependences of the upper critical fields $\mu_{0} H_{c 2}(T) \| a b$-plane and $\mu_{0} H_{c 2}(T) \| c$-axis near $T_{C}$ of this sample are shown in Fig. 4 by the dots. The theoretical 


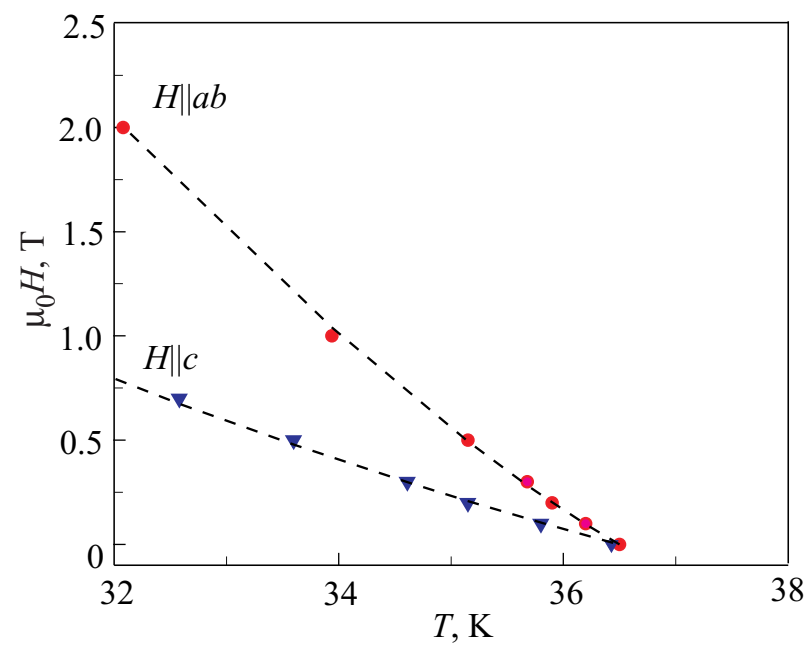

Fig. 4. The temperature dependence of the upper critical fields $\mu_{0} H_{c 2}(T) \| a b$-plane and $\mu_{0} H_{c 2}(T) \| c$-axis of the $\mathrm{MgB}_{2}$ film grown on the $c$-plane sapphire substrate with $T_{C}=36.5 \mathrm{~K}$. The experimental points are shown by the dots. The theoretical curves are shown by the dash line.

temperature dependences of the upper critical fields shown by the dash line in Fig. 4 were calculated using Eqs. (21) and (37) with parameters (44) and (45) calculated above. As we can see from this figure, the experimental points lie exactly on the respective theoretical curves.

Let us compare the theoretical results for the upper critical fields (Eqs. (21), (31), (37), and (42)) with experimental data taken from [42] for a $\mathrm{MgB}_{2}$ single crystal. The result of this comparison is presented in Fig. 5. The theoretical curve was plotted using the following parameters:

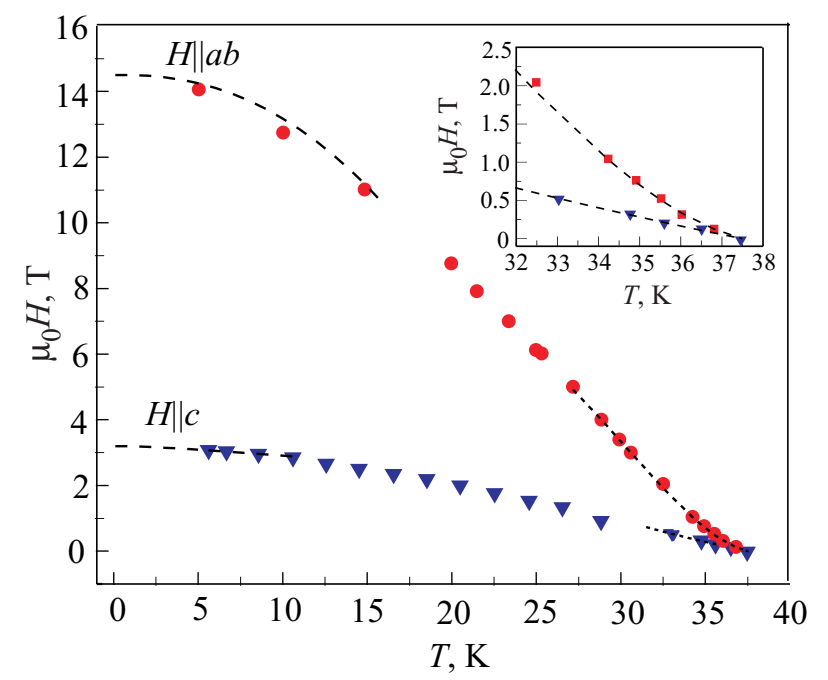

Fig. 5. The temperature dependence of the upper critical fields $\mu_{0} H_{c 2}(T) \| a b$-plane and $\mu_{0} H_{c 2}(T) \| c$-axis of the $\mathrm{MgB}_{2}$ single crystal with $T_{C}=37.5 \mathrm{~K}$ obtained from magnetization measurements [42] is shown by the dots. The theoretical curves are shown by the dash line. The range of temperatures near $T_{C}$ is enlarged in the inset.

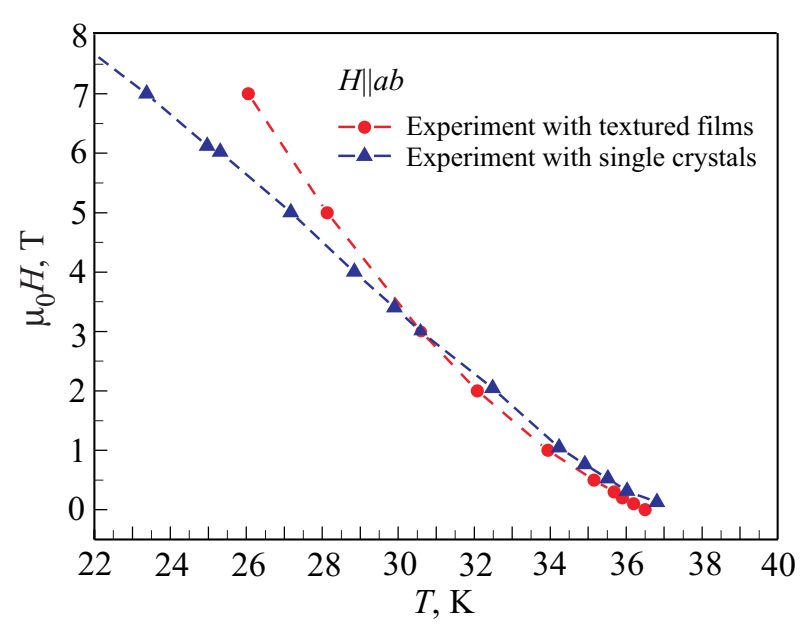

Fig. 6. Comparison of $\mu_{0} H_{c 2}(T) \| a b$-plane for the $c$-axis oriented $\mathrm{MgB}_{2}$ film with $T_{C}=36.5 \mathrm{~K}$ (shown by dots) with $\mu_{0} H_{c 2}(T) \| a b$ plane for the $\mathrm{MgB}_{2}$ single crystal [42] with $T_{C}=37.5 \mathrm{~K}$ (shown by triangles).

for Eq. (21): $\mu_{0} \mathfrak{x}_{1}=0.0036$ and $æ_{2}=20.78$;

for Eq. (31): $\mu_{0} H_{c 2}(0)=14.5 \mathrm{~T}, d_{1}=1.9, d_{2}=0.45$;

for Eq. (37): $\mu_{0} A_{1}=4$ and $\mu_{0} A_{1}=3.5$;

for Eq. (42): $c_{1}=0.012, c_{2}=-0.954$,

and $\mu_{0} H_{c 2}(0)=3.2 \mathrm{~T}$.

All these parameters were calculated from experimental data in the similar manner as described above for the $\mathrm{MgB}_{2}$ film.

As one can see from Fig. 5, there is also a good coincidence of the theoretical curves and the experimental results.

Comparing the theory parameters calculated for the $c$ axis oriented $\mathrm{MgB}_{2}$ film (Fig. 4) and the $\mathrm{MgB}_{2}$ single crystal (Fig. 5) we can see that these values are slightly different. This can be caused by the difference of their critical temperatures. Despite the differences, all experimental

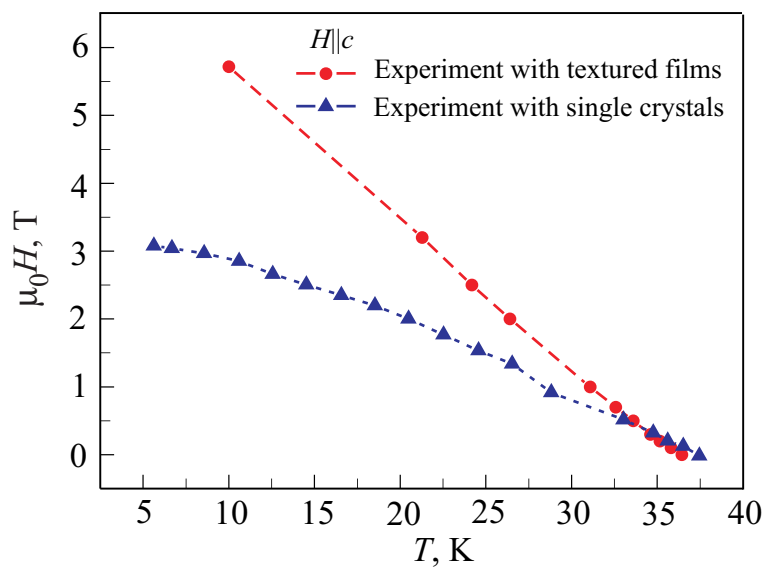

Fig. 7. Comparison of $\mu_{0} H_{c 2}(T) \| c$-axis measured for the $c$-axis oriented $\mathrm{MgB}_{2}$ film with $T_{C}=36.5 \mathrm{~K}$ (shown by dots) with $\mu_{0} H_{c 2}(T) \| c$-axis measured for the $\mathrm{MgB}_{2}$ single crystal [42] with $T_{C}=37.5 \mathrm{~K}$ (shown by triangles). 
curves are in analytical agreement with the theoretical description of Eqs. (21), (31), (37), and (42).

Note that the values of the upper critical fields obtained from the magnetization measurements of $\mathrm{MgB}_{2}$ single crystals are approximately the same as the respective values obtained from our resistive measurements of textured $\mathrm{MgB}_{2}$ films only near $T_{c}$. In the range of high fields and low temperatures the values of the upper critical fields of films are higher than the respective values of single crystals. This is illustrated in Figs. 6 and 7. We can see from Fig. 6 that the $H_{c 2}(a b)$ values of the $c$-axis oriented $\mathrm{MgB}_{2}$ film are higher at temperatures $T<30.5 \mathrm{~K}\left(\right.$ at $\left.\mu_{0} H>3 \mathrm{~T}\right)$. One can see from Fig. 7 that $H_{c 2}(c)$ of films is a linear function of temperature in the range of low temperatures: $T<33 \mathrm{~K}\left(\right.$ at $\left.\mu_{0} H>1 \mathrm{~T}\right)$. These facts can be caused by size effects in textured $\mathrm{MgB}_{2}$ films.

\section{Conclusions}

Main theoretical results of the microscopic two-band theory for the temperature dependence of the upper critical fields $H_{c 2}(a b)$ and $H_{c 2}(c)$ in a pure two-band superconductor with a band structure of the $\mathrm{MgB}_{2}$ type have been presented. The analytical expressions for $H_{c 2}(a b)$ and $H_{c 2}(c)$ at low temperatures and near the superconducting transition temperature have been calculated. We have also carried out experimental studies of the superconducting properties of pure textured $\mathrm{MgB}_{2}$ films in an external magnetic field; namely, the upper critical field parallel to the $c$-axis and the upper critical field parallel to the $a b$-plane of $\mathrm{MgB}_{2}$ films grown on the $c$-plane sapphire substrates as function of temperature have been measured and compared with the corresponding theoretical Eqs. (21) and (37). All results of the microscopic theory (formulas Eqs. (21), (31), (37), and (42)) were compared with earlier published experimental data of other authors. For this purpose, we developed a method to transform the analytical solutions of the microscopic theory equations for the upper critical field near the superconducting transition temperature and near the zero temperature for the two main crystallographic directions (see 2.2 and 2.3) to a convenient form for comparison with experimental data. In the analytical formulas, all parameters of the microscopic theory were isolated in separate terms and expressed then through several effective coefficients which are composite functions of them. These effective coefficients can be easily calculated using an appropriate number of experimental points. This method allows obtaining an accurate match between theory and experiment. So, Eqs. (21), (31), (37), and (42) have a very simple form (they contain several effective temperature independent coefficients) and we can easily determine these coefficients from experiment. If the values of the theory parameters (for instance, the Fermi velocities of the electrons or constants of the electron-phonon interactions) were determined each separately either from calculations or from the comparison with other experiments on $\mathrm{MgB}_{2}$, we would have a qualitative agreement between theory and experiment; this can be explained, in particular, by inexact determination of the theory parameters.

We have also demonstrated in our experiments that in high quality textured $\mathrm{MgB}_{2}$ films there is no broadening of the superconducting transition even in high fields.

We have observed that in the range of $T<33 \mathrm{~K}$ (at $\mu_{0} H>1 \mathrm{~T}$ ) the values of $H_{c 2}(c)$ for $c$-axis oriented $\mathrm{MgB}_{2}$ films are higher than the respective values for $\mathrm{MgB}_{2}$ single crystals; $H_{c 2}(c)$ for films is a linear function of temperature in the range of low temperatures. The $H_{c 2}(a b)$ values of $c$-axis oriented $\mathrm{MgB}_{2}$ films coincide with the respective values of $\mathrm{MgB}_{2}$ single crystals in the range of temperatures $T>30.5 \mathrm{~K}$ (at $\mu_{0} \mathrm{H}<3 \mathrm{~T}$ ) and they are higher at lower temperatures (at $\mu_{0} H>3 \mathrm{~T}$ ).

All graphs presented in this work have a positive curvature near the superconducting transition temperature. This effect is caused by the overlap between two energy bands on the Fermi surface in $\mathrm{MgB}_{2}$.

The studies carried out in this work confirm that the microscopic two-band model describes not only the thermodynamics but also the magnetic properties of the twoband systems like $\mathrm{MgB}_{2}$.

It is a pleasure to thank V. Smyslov, V. Yakunin, L. Konopko, A. Rusu, Th. Koch, Th. Schimmel, and A. Wixforth for continued support, encouragement, and fruitful discussion.

1. V.A. Moskalenko, Preprint (1958); Fiz. Met. Metalloved. 8, 503 (1959); Phys. Met. Metalog. 8, 25 (1959).

2. H. Suhl, B.T. Matthias, and L.R. Walker, Phys. Rev. Lett. 3, 552 (1959).

3. V.A. Moskalenko, L.Z. Kon, M.E. Palistrant, Low-Temperature Properties of Metals with Band - Spectrum Singularities, Stiinta, Kishinev (1989) [in Russian].

4. V.A. Moscalenko, L.Z. Kon, and M.E. Palistrant, The Theory of the Multiband Superconductivity, Tehnica, Bucharest (2008) [in Romanian]; http://www.theory//nipno.ro/ V.Barsan/ebooks/ Mosc.-2008. pdf [in English].

5. V.A. Moskalenko, Electromagnetic and Kinetic Properties of Superconducting Alloys with the Overlapping Energy Bands, Stiinta, Kishinev (1976) [in Russian].

6. M.E. Palistrant and M. Calalb, The Theory of the HighTemperature Superconductivity in Multiband Systems, Chisinau (2007) [in English].

7. V.A. Moskalenko and M.E. Palistrant, Statistical Physics and Quantum Field Theory, Nauka, Moscow (1973), p. 262 [in Russian].

8. V.A. Moskalenko, M.E. Palistrant, and V.M. Vakalyuk, Usp. Fiz. Nauk 161, 155 (1991) [Sov. Phys. Usp. 34, 717 (1991); arXiv: cond-mat / 03099671].

9. M.E. Palistrant, Int. J. Mod. Phys. 19, 929 (2005).

10. M.E. Palistrant, Moldavian J. Phys. Sci. 3, 133 (2004); arXiv:cond-mat/0305496 (2003). 
11. M.E. Palistrant, Condens. Matter Phys. 12, 677 (2009).

12. M.E. Palistrant and L.Z. Kon, Ukr. J. Phys. 55, 44 (2010).

13. B.T. Gelikman, Usp. Fiz. Nauk 88, 327 (1966).

14. B.T. Gelikman and V.Z. Kressin, Usp. Fiz. Nauk 99, 51 (1969).

15. V.M. Loktev, R.M. Quik, and S.G. Sharapov, Phys. Rep. 49, 1 (2001).

16. F. Bouqet, I. Wang, I. Sheikin, P. Toulemonde, M. Eisterer, H.W. Weber, S. Lee, S. Tajima, and A. Junod, Physica C385, 192 (2003); cond-mat/0210706.

17. P.C. Confield, S.I. Bud'ko, and D.K. Finemore, Physica C385, 1 (2003).

18. J. Nicol and J.P. Carbotte, Phys. Rev. B71, 054501 (2005).

19. M.E. Palistrant and V.A. Ursu, Zh. Eksp. Teor. Fiz. 131, 59 (2007); J. Exp. Theor. Phys. 104, 51 (2007).

20. M.E. Palistrant and V.A. Ursu, J. Supercond. Nov. Magn. 21, 171 (2008).

21. M.E. Palistrant, V.A. Moskalenko, and V.I. Dediu, Phys. Lett. 26A, 196 (1968).

22. M.E. Palistrant and V.I. Dediu, Researches under the Quantum Theory of Systems of Many Particles, RIO, Kishinev, (1969).

23. T. Dahma and N. Schopohe, Phys. Rev. Lett. 91, 017001 (2003).

24. P. Miranovic, K. Machita, and V.G. Kogan, J. Phys. Soc. Jpn. 72, 221 (2003).

25. V.H. Dao and M.E. Zhitomirsky, Eur. Phys. J. B44, 183 (2005).

26. I.N. Askerzade, Usp. Fiz. Nauk 176, 1025 (2006).

27. C.T. Rieck, R. Scharnberg, and N. Schopohl, J. Low Temp. Phys. 84, 981 (1991).

28. L.N. Gor'kov, Zh. Eksp. Teor. Fiz. 97, 833 (1959).
29. K. Maki and T. Tsuzuki, Phys. Rev. A139, 868 (1965).

30. V.A. Moskalenko, Zh. Exp. Theor. Phys. 51, 1163 (1966).

31. B.T. Gelikman, R.O. Zaitsev, and V.Z. Kresin, Sov. Phys. Solid State 9, 642 (1967); Fiz. Met. Metalloved. 23, 796 (1967).

32. M.E. Palistrant, V. Ursu, and A.V. Palistrant, Moldavian J. Phys. Sci. 4, 1 (2005).

33. V.A. Moskalenko, M.E. Palistrant, and V.A. Ursu, Theor. Math. Phys. 154, 94 (2008).

34. M.E. Palistrant, I.D. Cebotar, and V.A. Ursu, Moldavian J. Phys. Sci. 7, 292 (2008).

35. M.E. Palistrant, I.D. Cebotar, and V.A. Ursu, Zh. Exp. Theor. Phys. 109, 227 (2009).

36. M.E. Palistrant, J. Supercond. Nov. Magn. 23, 1427 (2010).

37. S.D. Bu, D.M. Kim, J.H. Choi, J. Giencke, E.E. Hellstrom, D.C. Larbalestier, S. Patnaik, L. Cooley, and C.B. Eom, J. Lettieri, D.G. Schlom, W. Tian, and X.Q. Pan, Appl. Phys. Lett. 81, 1851 (2002).

38. JCPDS-ICDD 46, 1212 (1997); ibid. 38, 1369 (1997).

39. X-ray Powder Data File, 45-0946, 38-1369, ASTM Spec. Tech. Publ. 48L, Inorganic, J.V. Smith (ed.), ASTM, Philadelphia, PA (1962).

40. A. Sidorenko, V. Zdravkov, V. Ryazanov, S. Horn, S. Klimm, R. Tidecks, A. Wixforth, Th. Koch, and Th. Schimmel, Philos. Mag. B85, 1783 (2005).

41. V.P.S. Awana A. Vajpayee, M. Mudgel, R. Rawat, S. Acharya, H. Kishan, E. Takayama-Muromachi, A.V. Narlikar, and I. Felner, Physica C467, 67 (2007).

42. M. Zehetmayer, M. Eisterer, H.W. Weber, J. Jun, S.M. Kazakov, J. Karpinski, and A. Wisniewski, Phys. Rev. B66, 052505 (2002). 